\title{
Milestones in Molecular Dynamics Simulations of Single-Walled Carbon Nanotube Formation: A Brief Critical Review
}

\author{
Stephan Irle ${ }^{1,2}(\bowtie)$, Yasuhito Ohta ${ }^{1, \uparrow}$, Yoshiko Okamoto, , Alister J. Page', Ying Wang ${ }^{2}$, and Keiji Morokuma ${ }^{1,3}(\bowtie)$ \\ ${ }^{1}$ Fukui Institute for Fundamental Chemistry, Kyoto University, Kyoto 606-8103, Japan \\ ${ }^{2}$ Institute for Advanced Research and Department of Chemistry, Nagoya University, Nagoya 464-8602, Japan \\ ${ }^{3}$ Cherry L. Emerson Center for Scientific Computation and Department of Chemistry, Emory University, Atlanta, GA 30322, USA \\ † Current address: Department of Chemistry, Nara Women's University, Nara 630-8506, Japan \\ Received: 17 April 2009 / Revised: 11 August 2009 / Accepted: 11 August 2009 \\ CTsinghua University Press and Springer-Verlag 2009. This article is published with open access at Springerlink.com
}

\begin{abstract}
We present a brief review of the most important efforts aimed at simulating single-walled carbon nanotube (SWNT) nucleation and growth processes using molecular dynamics (MD) techniques reported in the literature. MD simulations allow the spatio-temporal movement of atoms during nonequilibrium growth to be followed. Thus, it is hoped that a successful MD simulation of the entire SWNT formation process will assist in the design of chirality-specific SWNT synthesis techniques. We give special consideration to the role of the metal catalyst particles assumed in standard theories of SWNT formation, and describe the actual metal behavior observed in the reported MD simulations, including our own recent quantum chemical MD simulations. It is concluded that the use of a quantum potential is essential for a qualitatively correct description of the catalytic behavior of the metal cluster, and that carbide formation does not seem to be a necessary requirement for nucleation and growth of SWNTs according to our most recent quantum chemical MD simulations.
\end{abstract}

\section{KEYWORDS}

Carbon nanotubes, transition metal catalysis, molecular dynamics, reactive force fields, quantum chemistry

\section{Introduction}

Quasi-one-dimensional single-walled carbon nanotubes (SWNTs) [1, 2], together with quasizero-dimensional fullerenes [3] and quasi-twodimensional graphenes [4], are archetypal novel functional nanomaterials that can be tailored to specific needs. Their outstanding physicochemical properties [5], which originate from unique structural and related electronic features, have motivated the exploration of their applications in many different technological areas where nanometer scale structures are required $[6,7]$. In order for SWNTs to become routinely applicable in reality, however, explicit control over SWNT shape, size, density, and quality during synthesis must be attained. To date, such control has been elusive and remains the "holy grail" of SWNT synthesis research (see Sir Harry Kroto's comments in a recent interview in this respect [8]).

Until recently, SWNTs were commonly

Address correspondence to Stephan Irle, sirle@iar.nagoya-u.ac.jp; Keiji Morokuma, morokuma@fukui.kyoto-u.ac.jp 
synthesized using typical transition metals such as $\mathrm{Fe}, \mathrm{Co}, \mathrm{Ni}$, and Mo (or binary/ternary compounds thereof) as catalysts to promote the yield of synthesized SWNTs [9-11], but other metals such as $\mathrm{Mg}, \mathrm{Ca}, \mathrm{Ir}, \mathrm{W}$, and so on, have also shown such promotion [12]. For SWNT growth on nm-size particles of $\mathrm{Au}, \mathrm{Ag}$, and $\mathrm{Ni}$, Takagi et al. demonstrated that the size of the catalyst particles plays a crucial role in determining their catalytic activity [13]. Since the earliest days of SWNT research, the metal catalysts were believed to play essential roles in the nucleation and continued growth processes of SWNTs at high temperature [9, 14, 15]. Metal-free SWNT growth has not been achieved until rather recently [1619], with earlier efforts in this direction presumably being discouraged by the observation that noncatalytic carbon-arc synthetic routes had led only to multiwalled carbon nanotubes (MWNTs) [20], which are assumed to crystallize in supercooled liquid carbon droplets [11]. The addition of transition metals appeared therefore to be the "secret ingredient", creating the desired single layer tube structures [1], albeit without precise knowledge of how this feat is achieved. Thus, the nature of the various interactions of the metal catalyst with feedstock carbon or carbon precursors, such as CO [21], hydrocarbons [22, 23], and alcohols [24], has been a hotly debated topic in the discussion of the metal-catalyzed SWNT growth mechanism.

Currently, the most accepted general outline of the SWNT growth mechanism is provided by the popular vapor-liquid-solid (VLS) model [11]. In this model, the first step involves the generation of free carbon on the metal surface, which then dissolves into the metal catalyst itself. The resultant metal carbide, ultimately supersaturated with carbon, then facilitates the precipitation of carbon onto the surface of the metal catalyst. The outer carbon layer initiates the formation of an $\mathrm{sp}^{2}$ carbon cap nucleus on the metal surface. Growth into the capped tubular structure of the SWNT then ensues. The $(n, m)$ chirality is often assumed to be decided at the stage of cap formation $[25,26]$. Although the VLS model reasonably explains the phase separation dynamics between carbon and iron, it is still controversial whether SWNT growth always requires the formation of metal carbide or not. Indeed, it is likely that this depends on the type of the metal catalyst employed (see for instance two environmental transmission electron microscopy (ETEM) studies where cap nucleation was observed, one where bulk iron carbide was formed [27] and another where nickel did not form carbide in bulk [28]). Since the VLS model is a concept mainly based on thermodynamic reasoning, it does not promote per se an understanding of the atomistic details of the SWNT nucleation and growth processes. The underlying atomic-level reaction dynamics that drive the generation of carbon feedstock and the formation of the carbon cap precursor still remain unclear. Additionally, the exact role of the transition metal catalyst particle with respect to SWNT synthesis is still the subject of debate. Molecular dynamics (MD) approaches; however, allow the history of the carbon feedstock to be observed as it lands on the metal particle, decomposes (possibly forming a surface carbide), and ultimately recomposes forming an SWNT structure possessing specific $(n, m)$ chirality and diameter. MD investigations of SWNT growth and nucleation will therefore lead the way towards systematic improvements in our understanding of how greater control during SWNT synthesis may be attained. Thus, we need to be able to describe SWNT emergence atom by atom, and only time-resolved, atomistic MD simulations are at present in the position to tell the history of these atomic motions and reactions.

There is a strong sense that nonequilibrium SWNT self-assembly follows similar routes to those of emergent processes in the biosphere. Iijima has reportedly referred to the researchers growing SWNTs in their labs as "SWNT farmers" [29]. In the following section, we will trace back selected stories of "theoretical SWNT farmers" who synthesized their SWNTs either by using thought experiments or via computer MD simulations one nanotube at a time. We will cover the early ideas concerning the role of the metal catalyst, and discuss reactive empirical bond order (REBO) [30-32] -based simulations and the very rare (because computationally extremely expensive) Car-Parrinello density functional theory (DFT) MD (CPMD) simulations of SWNT growth [33]. Finally, we will summarize our own recent 
density-functional tight-binding-based MD (DFTB / MD) simulations of SWNT growth and nucleation $[34,35]$. We conclude this review by placing these simulations in a perspective regarding their adequacy to qualitatively describe the activities of the metal catalyst, and provide an outlook toward the future of MD simulations of SWNT formation. Due to the scope of the relevant literature concerning MD, static quantum chemical, and Monte Carlo investigations of SWNT growth, it is impossible for a single review to be exhaustive. We apologize for omitting a vast number of theoretical studies concerning SWNT growth from this review. We will give a more thorough review on another occasion.

\section{Early hypotheses on the role of the metal catalyst during SWNT formation}

To begin, we will mention the "scooter mechanism" introduced in Ref. [14]. Although the tight binding calculations reported in that work did not constitute MD simulations, the idea of keeping the growing SWNT edge from closing into a fullerene cage by a single nickel or cobalt atom scooting around on the open edge is truly a very dynamic one (refer to their key structure of an open-ended $(10,10)$ SWNT with a single $\mathrm{Ni}$ or $\mathrm{Co}$ atom chemisorbed onto its edge in Fig. 5 of Ref. [14]). Smalley and coworkers reasoned that the role of the "few metal atoms" must entail the prevention of pentagonal ring formation by way of annealing pentagons or other high-energy local defects at the time of their formation. Their calculations of barrier reductions for the annealing process by the presence of a single metal atom supported this hypothesis. In 1996, the Smalley group, using TEM experiments, also discovered that the Boudouard reaction $\left(2 \mathrm{CO} \rightarrow \mathrm{C}+\mathrm{CO}_{2}\right)$ could be catalyzed by preformed molybdenum particles of nanometer dimensions, leading to substantial SWNT growth (see Fig. 2 in Ref. [36]). This led to their hypothesis of a "yarmulke" (Yiddish for skullcap) mechanism, in which the growing $\mathrm{sp}^{2}$ carbon network avoids terminal unfilled valences by saturating these dangling bonds with surface defects of the metal cluster. The latter also catalyzes carbon feedstock creation from incoming $\mathrm{CO}$ molecules. Later observations by Yudasaka et al., who employed laser evaporation experiments with several laser target materials [15], indicated that the metal particles are in fact molten carbide droplets, which segregate carbon during cooling. At the same time it became commonplace in the catalytic chemical vapor decomposition (CCVD) community to discriminate between "root (or base) growth" $[23,37]$, in which the metal particles remain on a substrate, and "tip growth" [23], in which the metal particle is not anchored on the substrate surface. If no substrate is interacting with the catalyst or nanotube, the situation corresponds to a "floating catalyst", and it is this situation that is most often modeled in MD simulations of SWNT nucleation and growth.

At this point, we would like to reiterate the four "tasks" attributed to the metal catalyst that lead to SWNT formation, as opposed to fullerene cage formation or metal-free MWNT growth:

(1) a) Annealing (repairing) of defects such as pentagons, producing an all-hexagon $\mathrm{sp}^{2}$ carbon network due to the volatility of the metal-carbon bonds, and b) The prevention of SWNT closure.

(2) Saturation of terminal dangling bonds in the growing $\mathrm{sp}^{2}$ carbon structure.

(3) Catalyzing conversion of the carbon-containing feedstock into carbon in CCVD process.

(4) Providing critical carbon density for $\mathrm{sp}^{2}$ carbon "island" or "patch" nucleation by formation of metal droplets that contain carbon on the surface or in the bulk.

\section{Milestones in MD simulations of SWNT formation}

As we revisit the milestones in MD simulations of SWNT nucleation and growth, the degree to which each investigation satisfies each of these four hypothetical "tasks" will be emphasized. We will use Table 1 to keep track of these findings.

\subsection{REBO-based MD simulations}

The Tersoff potential $[38,39]$ and its application to hydrocarbon systems, called the REBO potential [30 -32], describes bond breaking/formation using a distance-dependent many-body bond order term and 
Table 1 Hypothetical metal catalyst tasks addressed in respective MD simulations. Task 1a: annealing (repairing) of defects such as pentagons yielding an all-hexagon $\mathrm{sp}^{2}$-carbon network due to the volatility of the metal-carbon bonds. Task $1 \mathrm{~b}$ : prevention of SWNT closure. Task 2: saturation of edge dangling bonds of the growing $\mathrm{sp}^{2}$ carbon structure. Task 3: catalyze carbon feedstock creation in CCVD processes. Task 4: provide critical carbon density for $\mathrm{sp}^{2}$ carbon "island" or "patch" nucleation by formation of metal carbide droplets

\begin{tabular}{|c|c|c|c|c|c|}
\hline \multirow{2}{*}{ Ref. } & \multicolumn{5}{|c|}{ Task } \\
\hline & $1 a$ & $1 b$ & 2 & 3 & 4 \\
\hline \multicolumn{6}{|c|}{ REBO-based MD } \\
\hline 43 & & $\sqrt{ }$ & & & \\
\hline 44 & & & $\sqrt{ }$ & & $\sqrt{ }$ \\
\hline $46,47,48,49,52$ & & $\sqrt{ }$ & $\sqrt{ }$ & & $\sqrt{ }$ \\
\hline \multicolumn{6}{|c|}{ CPMD } \\
\hline 37,53 & & & & & \\
\hline \multicolumn{6}{|c|}{ DFTB/MD } \\
\hline 59,62 & $(\sqrt{ })$ & $\sqrt{ }$ & $\sqrt{ }$ & & \\
\hline 63 & $\sqrt{ }$ & $\sqrt{ }$ & $\sqrt{ }$ & & \\
\hline
\end{tabular}

correctly dissociating attractive/repulsive diatomic potentials. This approach is nevertheless based on a local molecular mechanics approach, and therefore lacks explicit descriptions of quantum phenomena and Coulomb/van der Waals interactions. Hence, changes in the electronic structure of a dynamic, molecular system are not described. In particular, neither the evolution of $\pi$-conjugation and aromatic stabilization of carbons (important for $\mathrm{sp}^{2}$-hybridized carbon), nor charge transfer effects and the neardegeneracy of metal d-orbitals (important for the catalyst) can be captured by MD simulations based on the Tersoff and REBO potentials. Besides these grave deficiencies, the standard REBO potential has several other well-known problems. For example, in modeling gas-phase carbon densities it overestimates the $\mathrm{sp}^{3}$ fraction compared to corresponding DFT simulations [40], while it underestimates the sp fraction [41] which is of paramount importance with respect to fullerene cage self-assembly [42]. Consequently, task $1 \mathrm{a}$ is never addressed in REBO-type MD simulations. Nevertheless, since performing full quantum chemical MD simulations over sufficiently long time periods is out of the question due to the high computational cost, REBObased trajectories provided at least a zeroth order approximation that was certainly better than doing nothing at all.

The first such REBO-based MD simulation of metal-carbon co-vaporization in carbon arc or laser evaporation experiments was performed by Shibuta and Maruyama in 2002 [43] for a carbonnickel system. In remarkable agreement with the prediction of the very simple scooter model [14], the investigators found that in simulations starting with all atom systems at $2000 \mathrm{~K}$ the presence of even a few nickel atoms prohibited the complete closure of the self-assembling carbon cages. Thus, task $1 \mathrm{~b}$ was successfully modeled, although the SWNTs produced in these simulations exhibited a large number of non-hexagonal defects. In a subsequent study, the same investigators replaced the atomized metal vapor with $\mathrm{Ni}_{32}, \mathrm{Ni}_{108}$, and $\mathrm{Ni}_{256}$, each of which they exposed to carbon atom vapor under otherwise similar conditions [44]. For this reason, they associated the latter simulations to the SWNT formation in CCVD experiments. Although carbon caps are clearly nucleated on the surface of the nickel clusters, continued growth of the cap into a tubular SWNT structure was not observed. This key finding is shown in Fig. 1, and was attributed to the prevalent encapsulation of the metal particle by carbon, preventing the incorporation of additional carbon into the $\mathrm{sp}^{2}$ carbon network. In these simulations only task 2 was successfully observed before encapsulation took place, after which the catalyst "died". More careful inspections of the encapsulated nickel cluster structures showed that a surface carbide layer had formed, in accord with task 4 [45].

In order to circumvent the difficulties with encapsulation from external carbon (and to account for the fact that iron performs better in CCVD SWNT synthesis compared to nickel, whereas the latter performs better in carbon arc and laser evaporation synthesis), Ding, Bolton, and Rosen performed a series of REBO-based MD simulations where iron particles were "injected" with carbon atoms [4650]. Obviously, this manner of carbon addition is only possible for "theoretical SWNT farmers". In Ref. [46], the investigators showed that SWNTs subsequently grew from these iron-carbide particles at temperatures between 800 and $1400 \mathrm{~K}$ (see Fig. 2), whereas graphene sheets encapsulated the particles 


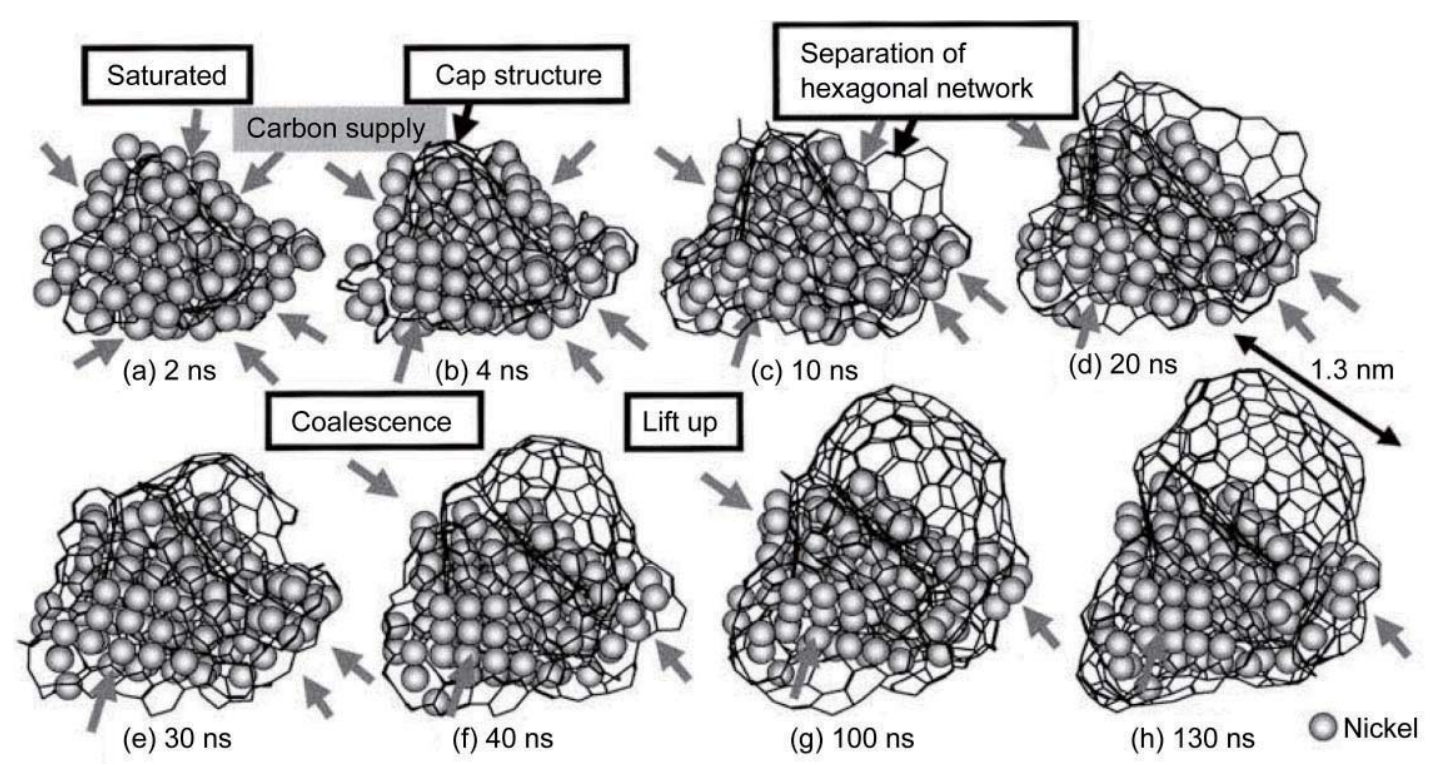

Figure 1 Snapshots of the metal-catalyzed growth process of a carbon cap structure: a 130-ns molecular dynamics calculation at $2500 \mathrm{~K}$ for $\mathrm{Ni}_{108}$. Gray circles represent nickel atoms and sticks show the developing sp ${ }^{2}$ carbon network on the surface of the nickel cluster. Carbon atoms surrounding the cluster were omitted for clarity. Gray arrows show a typical supply route of carbon atoms from exposed metal surface (Copyright Elsevier B.V. Reproduced from Ref. [44] with permission)

at temperatures below $600 \mathrm{~K}$. Three stages were identified during the nucleation of the $\mathrm{sp}^{2}$-carbon network: (1) at short time the iron-carbide particle was not saturated in carbon, and all carbon atoms were dissolved in the particle; (2) at intermediate time the iron-carbide cluster was highly supersaturated in carbon (task 4), and carbon strings, polygons and small graphitic islands started to nucleate on the cluster surface; (3) at longer time the iron-carbide cluster was supersaturated in carbon and, depending on the temperature, a graphene sheet, SWNT, or sootlike structure was formed (tasks $1 \mathrm{~b}$ and 2). At lower temperatures the kinetic energy was not sufficient to overcome the attractive forces between the particle and the graphitic islands. Consequently, because these islands could not lift off the particle, the iron particle became encapsulated. At temperatures above $800 \mathrm{~K}$ the kinetic energy was sufficiently high to overcome these attractive forces and so the graphitic island lifted off the particle, forming a cap. Between 800 and $1400 \mathrm{~K}$ these caps grew into defective SWNTs, and at temperatures larger than $1600 \mathrm{~K}$ the large number of defects in the growing carbon network produced a soot-like structure. The defects consisted of four-, five-, seven- and eight-membered rings that were frequently incorporated into the SWNT sidewalls and created an uneven $\mathrm{sp}^{2}$ carbon sidewall, preventing the tube from forming an identifiable $(n, m)$ chirality (failing task $1 \mathrm{a})$. The calculations also revealed that the growing SWNT maintains an open end on the cluster due to the strong bonding between the atoms at the open nanotube end and the cluster, in agreement with Smalley's idea of the yarmulke mechanism (task 2) [36]. It was observed that the number of defects in the SWNT structure could be reduced by lowering the rate of carbon addition to the iron-carbide particle, indicating that annealing plays a role in these SWNT formation simulations. One reason for the highly defective nanotubes obtained in these simulations is the lack of increasing $\pi$-conjugation stabilization with increasing tube length [51]. In Ref. [47], the investigators studied the effect of catalyst particle size (between 10 and 200 iron atoms) on the growth mechanism and resulting structure of SWNTs. The temperature for nanotube nucleation $(\sim 800-1100 \mathrm{~K})$ was chosen to be similar to that used in CCVD experiments, and the same VLS-type growth mechanism was observed for all cluster sizes studied. Catalyst particles containing at least 20 iron atoms nucleated SWNTs having better tubular structure than SWNTs nucleated from smaller clusters. In addition, the SWNTs that

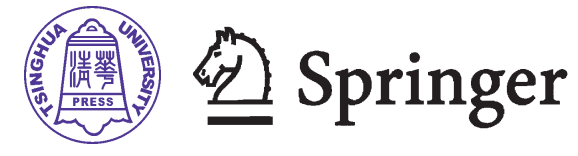


grew from the larger clusters had diameters that were similar to the cluster diameter, in agreement with experimental observations [23]. In Ref. [48], Ding, Rosen, and Bolton added the investigation of temperature and particle size interdependence to their previous studies, observing that smaller iron particles melt more readily at higher temperatures than larger particles. Concomitantly, it was observed that larger metal carbides formed more promptly at higher temperatures. In this respect it is interesting to note that a temperature gradient in the metal particle was found to be of less importance than the gradient of carbon concentration [49].

More recently, both the Bolton [50] and Maruyama [45] groups have studied the effect of the catalystsupport interactions on the catalyst melting behavior in attempts to model the CCVD SWNT growth process more realistically. Both groups have observed an inversely proportional relationship between the strength of the catalyst-support interaction and the tendency of the metal particle to liquefy. The Maruyama group have also compared the carbide formation tendencies of iron, cobalt, and nickel particles [52]. Using their own REBO parameters developed for these metal-carbon systems, these investigators have observed that cobalt interacts more strongly with the graphite lattice than does iron or nickel. Subsequently, Maruyama et al. [52] have

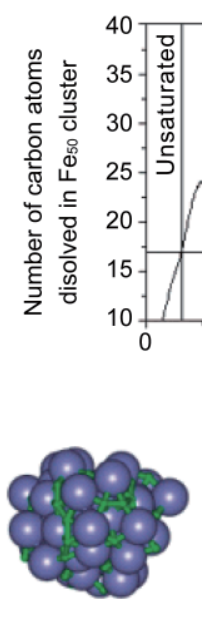

(a) $2.0 \mathrm{~ns}$
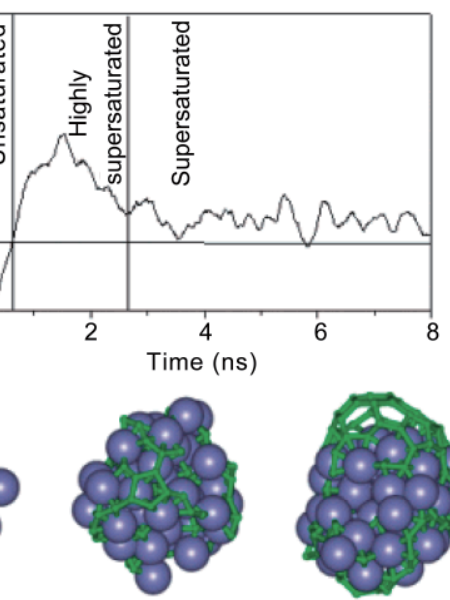

(b) $2.5 \mathrm{~ns}$

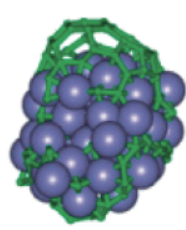

(c) $3 \mathrm{~ns}$

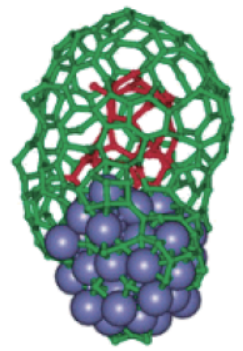

(d) $10 \mathrm{~ns}$
Figure 2 Snapshots during SWNT growth on an $\mathrm{Fe}_{50}$ cluster at $900 \mathrm{~K}$. One carbon atom is added to the center of the metal cluster every 40 ps. Iron and carbon atoms are represented using spheres and sticks, respectively (Copyright American Chemical Society. Reproduced from Ref. [46] with permission)

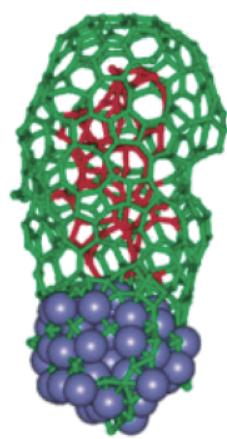

(e) $18 \mathrm{~ns}$ speculated that this stronger interaction could lead to more defective synthetic SWNTs.

\subsection{CPMD simulations}

CPMD simulations have been employed by two research groups for simulating the growth process of metal-catalyzed SWNT growth [37, 53]. Remarkably, the study by Gavillet et al. [37] was performed even before the first REBO-based MD simulations by the Maruyama group. As stated above, CPMD is a more accurate MD approach compared to REBObased MD, since it includes changes in the electronic structure throughout the dynamics. However, both reported CPMD studies $[37,53]$ were completely inadequate because of their short simulation time (due to high computational cost) and their unrealistic initial model geometries. Based on their limited simulation results, Gavillet et al. suggested that the segregation of carbon linear chains and atomic rings on the surface of a liquid-like cobaltcarbide particle represents the first stage of the cap nucleation process. In the same paper, Gavillet et al. also simulated the incorporation of five carbon atoms into a pre-assembled half-fullerene cap attached to a cobalt metal surface for 15 ps [37]. However, the use of unoptimized and unannealed initial starting structures in these simulations is likely to have caused dramatic, unrealistic changes in the movement of the carbon atoms. It is therefore questionable as to what conclusions can be drawn from the diffusion phenomenon that was observed in this single trajectory. In the simulations implemented by Raty and Galli [53], a nanodiamond fragment was used as an initial carbon complex, which for obvious reasons cannot be expected to appear under any SWNT synthesis conditions. This nanodiamond fragment, under the conditions of the study, converted itself rapidly into an $\mathrm{sp}^{2}$-network structure in the absence of a metal catalyst [54], 
a process which was described as "growth of carbon nanotubes" by these authors.

\subsection{DFTB-based MD simulations}

Neither REBO-based nor first principle-based MD simulations have succeeded in modeling the nucleation and sustained growth of a clean hexagononly SWNT from scratch as routinely achieved experimentally. This is despite the use of such tricks as injecting carbon atoms into the middle of the metal catalyst particle. It seems that one should "bite the bullet" and deal somehow with the complicated electronic structure of the evolving $\mathrm{sp}^{2}$-network and transition metal clusters at lower computational cost. The major obstacle for such a "cheap" CPMD approach is the limited availability of metal parameters in conventional semiempirical quantum chemical methods. Most notable in this respect are the MNDO/d [55] or PM6 [56] codes. However, the metal parameters are typically designed for singlemetal atom systems and are not suitable for the treatment of metal nanoparticles.

The DFTB / MD approach [35], which is a quantum mechanics / molecular dynamics (QM/MD) technique based on the DFTB electronic structure method, can play a role to fill in a gap between classical and first principle MD simulations. The DFTB method is approximately two orders of magnitude faster than first principles DFT and therefore enables longer simulations and provides more adequate model systems for nonequilibrium dynamics of nanosized clusters with quantum mechanical treatment of electrons. In addition, metal-carbon parameters for DFTB have recently been developed by the Morokuma group [57]. A finite electronic temperature approach $[58,59]$ ensures the applicability of the DFTB/MD method for nanometer size metal particles with high electronic densities of states around the Fermi level, as it allows the occupancy of each molecular orbital to change smoothly from 2 to 0 depending on its orbital energy. This approach effectively incorporates the open shell nature of the system due to near-degeneracy of iron d-orbitals, as well as carbon dangling bonds. We have found that in the absence of electronic temperature the iron cluster is much less reactive. This is considered to be an artifact of the simulation of a near-closed shell electronic wavefunction in that case.

Initially, we employed a $(5,5)$ armchair SWNT fragment attached to an $\mathrm{Fe}_{38}$ cluster as a model system (see Fig. 3(a)) [59]. The open end of the SWNT seed was terminated by hydrogen atoms, whereas the other end was bound to surface iron atoms of the $\mathrm{Fe}_{38}$ cluster. The use of such an iron-terminated nanotube seed was motivated by the experiment performed by the Smalley team [60,61]. In their experiment, a short nanotube fragment with the desired $(n, m)$ chirality was first extracted from grown SWNTs using a chemical treatment, after which both ends of the short SWNT fragment were terminated by iron particles. With the supply of ethanol [60] or ethylene [61] gas, the short SWNT fragments were successfully enlarged, becoming longer nanotubes with the

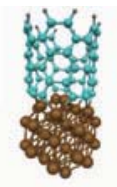

(a)

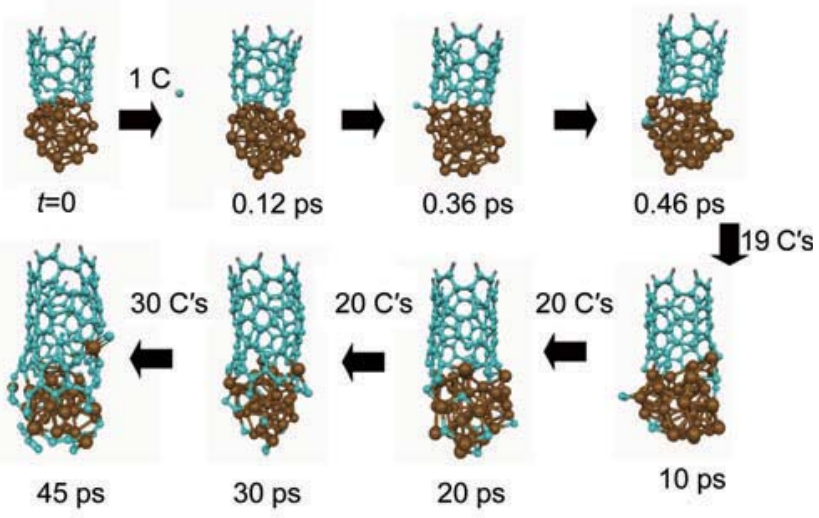

(b)

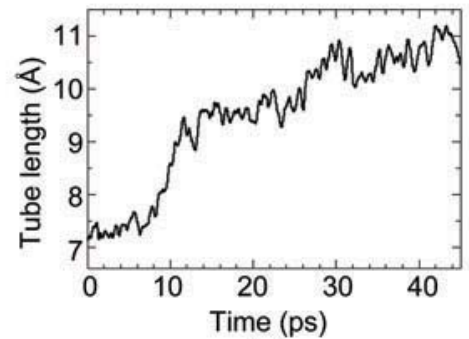

(c)

Figure 3 Fe-catalyzed continued (5,5)-SWNT growth simulations: (a) initial $\mathrm{H}_{10} \mathrm{C}_{60} \mathrm{Fe}_{38}$ structure where gray, cyan, and brown spheres represent hydrogen, carbon, and iron, respectively; (b) snapshots along the growth process of the SWNT; (c) time-variation of the tube length (Copyright American Chemical Society. Reproduced from Ref. [59] with permission) 
same diameter. Conversely, in the absence of iron particles, such growth was not observed, confirming the importance of the iron particle as a catalyst. This experiment therefore presented the possibility of chirality-controlled SWNT growth using such a cloning technique.

In our growth simulations [59], gas-phase carbon atoms were supplied around the boundary area between the nanotube and the iron cluster every $0.5 \mathrm{ps}$ in order to focus on reactivity of the metalcarbon interface of the nanotube-iron cluster. Figure 3(b) shows the growth process of an SWNT obtained by our carbon supply simulations. The supplied carbon atoms were promptly incorporated into the rim of the nanotube to form short polyyne chains. They then played a role as a linkage between the nanotube and iron surface. Frequent carbon-iron bond breaking and formation of the polyyne chains promoted the rearrangement of constituent atoms, forming new polygonal (pentagonal, hexagonal, and heptagonal) rings at the rim of the nanotube. The formation of these polygonal rings contributed to the construction of carbon sidewall. Lift-off and growth of the nanotube fragment on the iron cluster were subsequently observed during these carbonsupply simulations (see Fig. 3(c)). This was the first substantial growth simulation of metal-catalyzed SWNT by means of $\mathrm{QM} / \mathrm{MD}$ simulation reported in the literature, to the best of our knowledge. Tasks $1 \mathrm{~b}, 2$, and 4 were all reflected by the behavior of the metal cluster in our simulations. Task 1a (the annealing of non-hexagonal defects) was frequently observed, although the resulting structure still contained a substantial number of pentagons and hexagons (but no four- or eight-membered rings as observed in REBO-based MD simulations, see for instance Fig. 5 in Ref. [46]). Recent DFTB/MD simulations of SWNT growth performed in our group have since shown that the annealing of non-hexagonal permission) defects in the nanotube sidewall is in fact driven by the relative rates of defect addition and removal in the SWNT structure. Thus, annealing of defects (task 1a) during SWNT growth is enhanced using lower rates of carbon feedstock supply.

These successful simulations of SWNT growth motivated us to employ the rapid continued SWNT$\mathrm{Fe}_{38}$ growth methodology to a wider range of environmental temperatures from $1000 \mathrm{~K}$ to $2000 \mathrm{~K}$. The temperature dependence of continued growth of SWNTs was therefore investigated [62]. We have observed a variety of reaction processes between feedstock carbon atoms and the nanotube-metal cluster, depending on the nuclear temperature employed. The temperature dependence of the primary reaction dynamics was analyzed using growth rates and phenomenological observations. We found that relatively long polyyne chains tend to grow from the SWNT sidewall at the lower temperature of $1000 \mathrm{~K}$, while near structural collapse of the nanotube begins to occur at $2000 \mathrm{~K}$ (see Fig. 4). These features were attributed to inefficient growth at these temperatures under the given, otherwise identical, simulation conditions.

The rapid growth simulations provided meaningful information to understand the reactivity
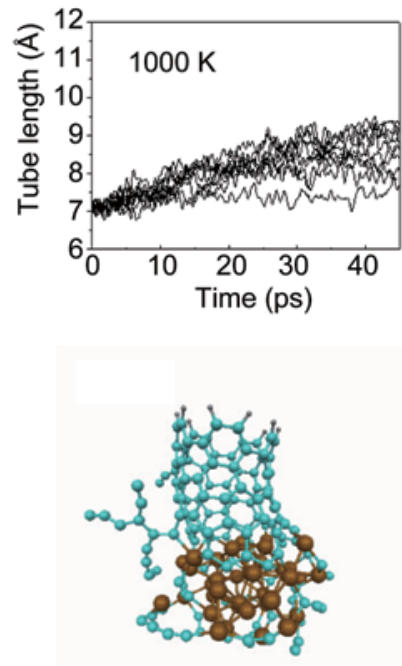

(a) $1000 \mathrm{~K}$
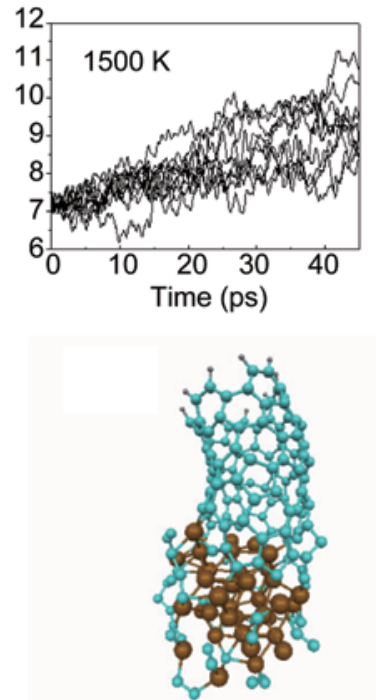

(b) $1500 \mathrm{~K}$
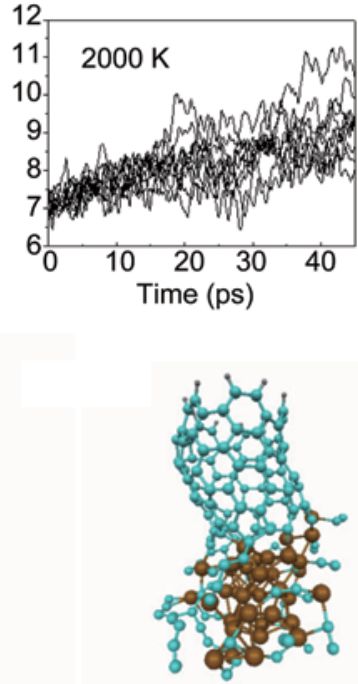

(c) $2000 \mathrm{~K}$
Figure 4 Time-variation of tube length evaluated from ten Fe-catalyzed continued $(5,5)-S W N T$ growth simulations trajectories at (a) $T=1000 \mathrm{~K}$, (b) $1500 \mathrm{~K}$, and (c) $2000 \mathrm{~K}$, and corresponding characteristic structures after 45 ps SWNT growth simulations. Definitions of colored spheres are the same as in Fig. 3 (Copyright American Chemical Society. Reproduced from Ref. [62] with 
of the nanotube-Fe interface. However, the feedstock gas-phase $\mathrm{C}$ atoms were forcibly supplied around nanotube-Fe interface during growth simulations and thereby the reaction area was rather biased to the nanotube-Fe interface. In addition, the highly exothermic reactions between $\mathrm{C}$ atoms and the nanotube-Fe interface instantaneously led to local heating causing overestimation of rearrangement of constituent atoms even though a thermostat was employed to maintain a target temperature in a canonical ensemble. For the nanotube-Fe cluster, the cross-section of the metal surface should be much larger than that of the nanotube-metal interface. It follows then that the majority of feedstock carbons would first bond to and diffuse over the metal surface, before being incorporated into the rim of the SWNT. For this reason, we subsequently investigated the effects of feedstock carbon diffusion of the iron cluster surface, using a different growth simulation methodology.

In order to implement continued growth simulations of SWNTs based on carbon diffusion on/inside an iron cluster, we have used a $\mathrm{C}_{40}$ carbon cap attached to an $\mathrm{Fe}_{38}$ cluster (See Fig. 5(a)) [63]. In this model, gas-phase carbon atoms were supplied around the surface of the iron cluster every $0.5 \mathrm{ps}$ for 30 ps. The resultant iron-carbon complex was then annealed for a further 160 ps without supplying further carbon feedstock. As shown in Fig. 5(b), we observed that surface carbon clusters were mainly incorporated into the rim of the carbon cap cluster through surface diffusion, and extended the carbon sidewall without dissolving into the $\mathrm{Fe}_{38}$ cluster. This indicates that iron carbide formation is not necessary for SWNT growth. From the ring condensation analysis of all trajectories obtained, we also found that newly created polygonal rings were largely pentagonal and hexagonal, with no long-lived heptagonal rings being observed (See Fig. 5(c)). This feature was in contrast to our previous carbon supply simulations, in which carbon feedstock was supplied near the nanotube-metal interface [59,62]. In the latter carbon-supply simulations, a considerable number of seven-membered rings were produced in the nanotube sidewall by rapid hexagon-toheptagon transformation induced by the reaction

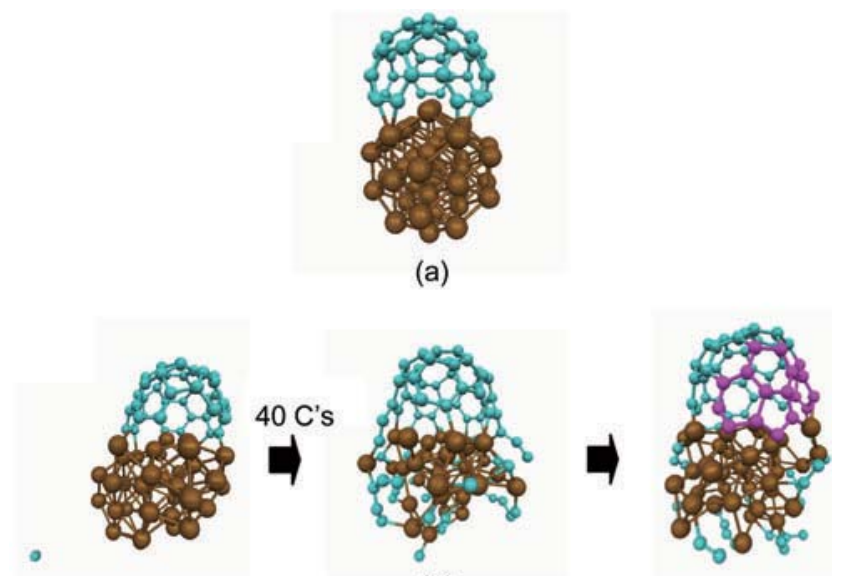

(b)

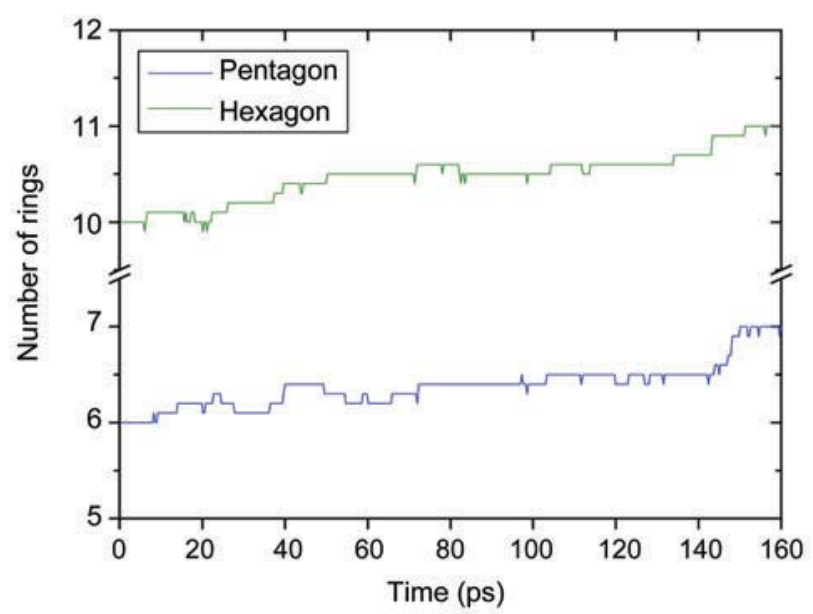

(c)

Figure 5 SWNT-growth simulations from a $\mathrm{C}_{40}$ carbon cap fragment on an $\mathrm{Fe}_{38}$ cluster. (a) Initial $\mathrm{C}_{40} \mathrm{Fe}_{38}$ structure. Definitions of colored spheres are the same as in Fig. 3. (b) Snapshots along the growth process of the SWNT. Pink spheres represent incorporated ironsurface carbon clusters at the rim of the $C_{40}$ cap fragment. (c) Timevariation of all pentagonal and hexagonal rings averaged over all ten diffusion trajectories (Copyright Elsevier B.V. Reproduced from Ref. [63] with permission)

between the incident carbon atoms and preexisting hexagonal rings. These differences observed between our carbon-supply and carbon-diffusion simulations highlight that surface diffusing carbons contribute to the construction of the nanotube sidewall producing fewer defects. The rim of the carbon cap is therefore a narrow key window for sidewall growth in the developing SWNT.

A certainly surprising finding from our recent DFTB/MD simulations [63] is that it does not seem necessary for a state of carbon supersaturation in the metal catalyst (task 4) to be achieved for continued SWNT growth to occur. Preliminary results also

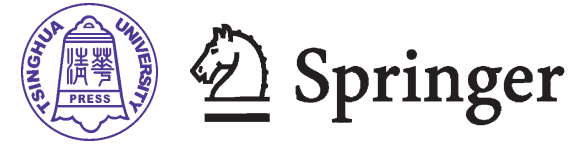


indicate that this supersaturation is also not necessary for the nucleation of a carbon cap structure itself. While this is a real observation, no inference as to the relative role of the supersaturated metal carbide (with respect to SWNT nucleation and growth) can be made from our simulations. That is, these results do not suggest that if a metal carbide happened to be formed at an earlier stage, it could not nucleate a carbon cap and perform continued SWNT growth. It is rather likely that surface carbide formation provides a critical carbon density on the metal surface to create enough polyynes that can nucleate to yield $\mathrm{sp}^{2}$ carbon ring systems. Further investigations into this question are currently ongoing in our group.

\section{Comparison of different SWNT growth MD simulation approaches and outlook}

It is apparent from Table 1 that the chief task of the metal cluster (viz. the annealing of non-hexagonal defects from the SWNT, thus providing an SWNT with a well-defined $(n, m)$ chirality, (task 1a)) cannot be achieved using REBO-based MD simulations. The effect of the increase of the $\pi$-conjugated electronic structure with increased stabilization is beyond the capability of simple many-body potentials, as discussed above. Further, the second chief objective of the metal cluster, namely keeping the carbon structure open for continued sidewall growth by incoming new carbon feedstock (task 1b), cannot be achieved without "cheating": that is, a supply of carbon from the outside of the metal cluster always results in complete encapsulation and "death of the catalyst". Thus, REBO-based MD simulations constitute a dead-end for the simulation of SWNT nucleation and growth, and have largely been abandoned by the groups of Maruyama and Bolton, the latter using ingenious ways to employ DFT calculations for the SWNT growth problem [64, 65]. Both chief tasks of the metal catalyst can, however, be modeled successfully using DFTB/MD simulations, particularly with lower carbon feeding rates in surface diffusion simulations such as that presented in Ref. [63]. CPMD simulations are too costly and cannot be employed at present for the simulation of SWNT nucleation and growth dynamics over meaningful timescales. Although we have not yet arrived at the goal of "farming" well-defined $(n, m)$ specific SWNTs from scratch in our computers, results discussed in this review indicate that this aim is well within the reach of future DFTB/MDsimulations, in particular when considering even lower carbon feedstock supply rates.

An issue that has not been addressed at all in any MD simulation of SWNT growth to date is the catalytic activity of the metal particles, producing carbon feedstock from precursor molecules such as acetylene. DFTB/MD simulations to this end are likewise ongoing and indicate the formation of polyacetylene chains as initial stages in the conversion of $\mathrm{C}_{2} \mathrm{H}_{2}$ molecules to pure carbon feedstock.

\section{Acknowledgements}

This work was in part supported by a Core Research for Evolutional Science and Technology (CREST) grant in the Area of High Performance Computing for Multi-Scale and Multi-Physics Phenomena from the Japan Science and Technology Agency (JST). One of the authors (SI) also acknowledges support by the Program for Improvement of Research Environment for Young Researchers from Special Coordination Funds for Promoting Science and Technology (SCF) commissioned by the Ministry of Education, Culture, Sports, Science and Technology (MEXT) of Japan. Our own simulations were performed in part using the computer resources at the Research Center for Computational Science (RCCS), Okazaki Research Facilities, National Institutes for Natural Sciences, and at the Academic Center for Computing and Media Studies (ACCMS) at Kyoto University.

\section{References}

[1] lijima, S.; Ichihashi, T. Single-shell carbon nanotubes of 1-nm diameter. Nature 1993, 363, 603-605.

[2] Bethune, D. S.; Kiang, C. H.; Devries, M. S.; Gorman, G.; Savoy, R.; Vazquez, J.; Beyers, R. Cobalt-catalyzed growth of carbon nanotubes with single-atomic-layer walls. Nature 1993, 363, 605-607.

[3] Kroto, H. W.; Heath, J. R.; O'Brien, S. C.; Curl, R. F.; 
Smalley, R. E. C 60 : Buckminsterfullerene. Nature 1985, 318, 162-163.

[4] Novoselov, K. S.; Geim, A. K.; Morozov, S. V.; Jiang, D.; Zhang, Y.; Dubonos, S. V.; Grivorieva, L. V.; Firsov, A. A. Electric field effect in atomically thin carbon films. Science 2004, 306, 666-669.

[5] Saito, R.; Dresselhaus, G.; Dresselhaus, M. S. Physical Properties of Carbon Nanotubes; Imperial College Press: London, 1998.

[6] Dresselhaus, M. S.; Dresselhaus, G.; Avouris, P. Carbon Nanotubes: Synthesis, Structure, Properties, and Applications; Springer: New York, 2001.

[7] Baughman, R. A.; Zakhidov, A. A.; de Heer, W. A. Carbon nanotubes - The route toward applications. Science 2002, 297, 787-792.

[8] Palmer, D. J. Where nano is going. Nano Today 2008, 3, 46-47.

[9] Journet, C.; Maser, W. K.; Bernier, P.; Loiseau, A.; Lamy de la Chapelle, M.; Lefrant, S.; Deniard, P.; Lee, R.; Fischer, J. E. Large-scale production of single-walled carbon nanotubes by the electric-arc technique. Nature 1997, 388, 756-758.

[10] Moisala, A.; Nasibulin, A. G.; Kauppinen, E. I. The role of metal nanoparticles in the catalytic production of singlewalled carbon nanotubes-A review. J. Phys. Cond. Matter 2003, 15, S3011-S3035.

[11] Harris, P. J. F. Solid state growth mechanisms for carbon nanotubes. Carbon 2007, 45, 229-239.

[12] Esconjauregui, S.; Whelan, C. M.; Maex, K. The reasons why metals catalyze the nucleation and growth of carbon nanotubes and other carbon nanomorphologies. Carbon 2009, 47, 659-669.

[13] Takagi, D.; Homma, Y.; Hibino, H.; Suzuki, S.; Kobayashi, Y. Single-walled carbon nanotube growth from highly activated metal nanoparticles. Nano Lett. 2006, 6, 2642 $-2645$.

[14] Thess, A.; Lee, R.; Nikolaev, P.; Dai, H. J.; Petit, P.; Robert, J.; Xu, C. H.; Lee, Y. H.; Kim, S. G.; Rinzler, A. G.; Colbert, D. T.; Scuseria, G. E.; Tomanek, D.; Fischer, J. E.; Smalley, R. E. Crystalline ropes of metallic carbon nanotubes. Science 1996, 273, 483-487.

[15] Yudasaka, M.; Yamada, R.; Sensui, N.; Wilkins, T.; Ichihashi, T.; lijima, S. Mechanism of the effect of NiCo, $\mathrm{Ni}$ and $\mathrm{Co}$ catalysts on the yield of single-wall carbon nanotubes formed by pulsed Nd:YAG laser ablation. J. Phys. Chem. B 1999, 103, 6224-6229.
[16] Kusunoki, M.; Suzuki, T.; Hirayama, M.; Shibata, N.; Kaneko, K. A formation mechanism of carbon nanotube films on SiC(0001). Appl. Phys. Lett. 2000, 77, 531-533.

[17] Takagi, D.; Hibino, H.; Suzuki, S.; Kobayashi, Y.; Homma, Y. Carbon nanotube growth from semiconductor nanoparticles. Nano Lett. 2007, 7, 2272-2275.

[18] Huang, S.; Cai, Q.; Chen, J.; Qian, Y.; Zhang, L. Metalcatalyst-free growth of single-walled carbon nanotubes on substrates. J. Am. Chem. Soc. 2009, 131, 20942095.

[19] Liu, B.; Ren, W.; Gao, L.; Li, S.; Pei, S.; Liu, C.; Jiang, C.; Cheng, H. M., Metal-catalyst-free growth of singlewalled carbon nanotubes. J. Am. Chem. Soc. 2009, 131, 2082-2083.

[20] lijima, S. Helical microtubules of graphitic carbon. Nature 1991, 354, 56-58.

[21] Nikolaev, P.; Bronikowski, M. J.; Bradley, R. K.; Rohmund, F.; Colbert, D. T.; Smith, K. A.; Smalley, R. E. Gas-phase catalytic growth of single-walled carbon nanotubes from carbon monoxide. Chem. Phys. Lett. 1999, 313, 91-97.

[22] Cheng, H. M.; Li, F.; Sun, X.; Brown, S. D. M.; Pimenta, M. A.; Marucci, A.; Dresselhaus, G.; Dresselhaus, M. S. Bulk morphology and diameter distribution of single-walled carbon nanotubes synthesized by catalytic decomposition of hydrocarbons. Chem. Phys. Lett. 1998, 289, 602-610.

[23] Cassell, A. M.; Raymakers, J. A.; Kong, J.; Dai, H. Large scale CVD synthesis of single-walled carbon nanotubes. J. Phys. Chem. B 1999, 103, 6484-6492.

[24] Maruyama, S.; Kojima, R.; Miyauchi, Y.; Chiashi, S.; Kohno, M. Low-temperature synthesis of high-purity single-walled carbon nanotubes from alcohol. Chem. Phys. Lett. 2002, 360, 229-234.

[25] Reich, S.; Li, L.; Robertson, J. Structure and formation energy of carbon nanotube caps. Phys. Rev. B 2005, 72, 165423.

[26] Gomez-Gualdron, D. A.; Balbuena, P. B. The role of cap chirality in the mechanism of growth of single-wall carbon nanotubes. Nanotechnology 2008, 19, 485604.

[27] Yoshida, H.; Takeda, S.; Uchiyama, T.; Kohno, H.; Homma, Y. Atomic-scale in situ observation of carbon nanotube growth from solid state iron carbide nanoparticles. Nano Lett. 2008, 8, 2082-2086.

[28] Hofmann, S.; Sharma, R.; Ducati, C.; Du, G.; Mattevi, C.; Cepek, C.; Cantoro, M.; Pisana, S.; Parvez, A.; Cervantes-Sodi, F.; Ferrari, A. C.; Dunin-Borkowski, R.; Lizzit, S.; Petaccia, L.; Goldoni, A.; Robertson, J. In situ 
observations of catalyst dynamics during surface-bound carbon nanotube nucleation. Nano Lett. 2007, 7, 602608.

[29] Jiang, K. Super-aligned carbon nanotube arrays: Controlled synthesis, physical properties and applications. IUMRS International Conference in Asia (IUMRS-ICA), Nagoya, Japan, 2008.

[30] Brenner, D. W. Empirical potential for hydrocarbons for use in simulating the chemical vapor-deposition of diamond films. Phys. Rev. B 1990, 42, 9458-9471.

[31] Brenner, D. W. Empirical potential for hydrocarbons for use in simulating the chemical vapor deposition of diamond films. [Erratum to document cited in CA114(6):53045x]. Phys. Rev. B 1992, 46, 1948.

[32] Brenner, D. W.; Shenderova, O. A.; Harrison, J. A.; Stuart, S. J.; Ni, B.; Sinnott, S. B. A second-generation reactive empirical bond order (REBO) potential energy expression for hydrocarbons. J. Phys. Cond. Matter 2002, 14, 783802.

[33] Car, R.; Parrinello, M. Unified approach for moleculardynamics and density-functional theory. Phys. Rev. Lett. 1985, 55, 2471-2474.

[34] Porezag, D.; Frauenheim, T.; Kohler, T.; Seifert, G.; Kaschner, R. Construction of tight-binding-like potentials on the basis of density-functional theory-Application to carbon. Phys. Rev. B 1995, 51, 12947-12957.

[35] Elstner, M.; Porezag, D.; Jungnickel, G.; Elsner, J.; Haugk, M.; Frauenheim, T.; Suhai, S.; Seifert, G. Self-consistentcharge density-functional tight-binding method for simulations of complex materials properties. Phys. Rev. $B$ 1998, 58, 7260-7268.

[36] Dai, H.; Rinzler, A. G.; Nikolaev, P.; Thess, A.; Colbert, D. T.; Smalley, R. E. Single-wall nanotubes produced by metal-catalyzed disproportionation of carbon monoxide. Chem. Phys. Lett. 1996, 260, 471-475.

[37] Gavillet, J.; Loiseau, A.; Journet, C.; Willaime, F.; Ducastelle, F.; Charlier, J. -C. Root-growth mechanism for single-wall carbon nanotubes. Phys. Rev. Lett. 2001, 87, 275504

[38] Tersoff, J. New empirical approach for the structure and energy of covalent systems. Phys. Rev. B 1988, 37, 6991 -7000 .

[39] Tersoff, J. Modeling solid-state chemistry: Interatomic potentials for multicomponent systems. Phys. Rev. $B$ 1989, 39, 5566-5568.

[40] Marks, N. A.; Cooper, N. C.; McKenzie, D. R.; McCulloch,
D. G.; Bath, P.; Russo, S. P. Comparison of densityfunctional, tight-binding, and empirical methods for the simulation of amorphous carbon. Phys. Rev. B 2002, 65, 075411.

[41] Zheng, G. S.; Irle, S.; Elstner, M.; Morokuma, K. Quantum chemical molecular dynamics model study of fullerene formation from open-ended carbon nanotubes. J. Phys. Chem. A 2004, 108, 3182-3194.

[42] Irle, S.; Zheng, G.; Wang, Z.; Morokuma, K. The $C_{60}$ formation puzzle "solved": QM/MD simulations reveal the shrinking hot giant road of the dynamic fullerene self-assembly mechanism. J. Phys. Chem. B 2006, 110, 14531-14545.

[43] Shibuta, Y.; Maruyama, S. Molecular dynamics simulation of generation process of SWNTs. Physica B 2002, 323, 187-189.

[44] Shibuta, Y.; Maruyama, S. Molecular dynamics simulation of formation process of single-walled carbon nanotubes by CCVD method. Chem. Phys. Lett. 2003, 382, 381386.

[45] Shibuta, Y.; Maruyama, S. A molecular dynamics study of the effect of a substrate on catalytic metal clusters in nucleation process of single-walled carbon nanotubes. Chem. Phys. Lett. 2007, 437, 218-223.

[46] Ding, F.; Bolton, K.; Rosen, A. Nucleation and growth of single-walled carbon nanotubes: A molecular dynamics study. J. Phys. Chem. B 2004, 108, 17369-17377.

[47] Ding, F.; Rosen, A.; Bolton, K. Molecular dynamics study of the catalyst particle size dependence on carbon nanotube growth. J. Chem. Phys. 2004, 121, 27752779.

[48] Ding, F.; Bolton, K.; Rosen, A. Iron-carbide cluster thermal dynamics for catalyzed carbon nanotube growth. J. Vac. Sci. Technol. A 2004, 22, 1471-1476.

[49] Ding, F.; Bolton, K.; Rosen, A. Molecular dynamics study of SWNT growth on catalyst particles without temperature gradients. Comput. Mater. Sci. 2006, 35, 243-246.

[50] Ding, F.; Rosen, A.; Curtarolo, S.; Bolton, K. Modeling the melting of supported clusters. Appl. Phys. Lett. 2006 88,133110 .

[51] Ding, F.; Larsson, P.; Larsson, J. A.; Ahuja, R.; Duan, H.; Rosen, A.; Bolton, K. The importance of strong carbonmetal adhesion for catalytic nucleation of single-walled carbon nanotubes. Nano Lett. 2008, 8, 463-468.

[52] Shibuta, Y.; Maruyama, S. Bond-order potential for 
transition metal carbide cluster for the growth simulation of a single-walled carbon nanotube. Comput. Mater. Sci. 2007, 39, 842-848.

[53] Raty, J. Y.; Gygi, F.; Galli, G. Growth of carbon nanotubes on metal nanoparticles: A microscopic mechanism from ab initio molecular dynamics simulations. Phys. Rev. Lett. 2005, 95, 096103.

[54] Raty, J. Y.; Galli, G.; Bostedt, C.; van Buuren, T. W.; Terminello, L. J. Quantum confinement and fullerenelike surface reconstructions in nanodiamonds. Phys. Rev. Lett. 2003, 90, 037401.

[55] Thiel, W.; Voityuk, A. A. Extension of the MNDO formalism to d orbitals: Integral approximations and preliminary numerical results. Theor. Chim. Acta 1996, 93, 315.

[56] Stewart, J. J. P. MOPAC2009, Cache Software Group: 2009.

[57] Zheng, G. S.; Witek, H. A.; Bobadova-Parvanova, P.; Irle, S.; Musaev, D. G.; Prabhakar, R.; Morokuma, K. Parameter calibration of transition-metal elements for the spin-polarized self-consistent-charge densityfunctional tight-binding (DFTB) method: Sc, Ti, Fe, Co, and Ni. J. Chem. Theory Comput. 2007, 3, 1349-1367.

[58] Weinert, M.; Davenport, J. W. Fractional occupations and density-functional energies and forces. Phys. Rev. $B$ 1992, 45, 13709-13712.

[59] Ohta, Y.; Irle, S.; Okamoto, Y.; Morokuma, K. Rapid growth of a single-walled carbon nanotube on an iron cluster: Density-functional tight-binding molecular dynamics simulations. ACS Nano 2008, 2, 1437-1444.

[60] Wang, Y.; Kim, M. J.; Shan, H.; Kittrell, C.; Fan, H.; Ericson, L. M.; Hwang, W.-F.; Arepalli, S.; Hauge, R. H.; Smalley, R. E. Continued growth of single-walled carbon nanotubes. Nano Lett. 2005, 5, 997-1002.

[61] Smalley, R. E.; Li, Y. B.; Moore, V. C.; Price, B. K.; Colorado, R.; Schmidt, H. K.; Hauge, R. H.; Barron, A. R.; Tour, J. M. Single wall carbon nanotube amplification: En route to a type-specific growth mechanism. J. Am. Chem. Soc. 2006, 128, 15824-15829.

[62] Ohta, Y.; Okamoto, Y.; Irle, S.; Morokuma, K. Temperature dependence of iron-catalyzed continued single-walled carbon nanotube growth rates: Density functional tight-binding molecular dynamics simulations. J. Phys. Chem. C 2009, 113, 159-169.

[63] Ohta, Y.; Okamoto, Y.; Irle, S.; Morokuma, K. Densityfunctional tight-binding molecular dynamics simulations of SWCNT growth by surface carbon diffusion on an iron cluster. Carbon 2009, 47, 1270-1275.

[64] Larsson, P.; Larsson, J. A.; Ahuja, R.; Ding, F.; Yakobson, B. I.; Duan, H. M.; Rosen, A.; Bolton, K. Calculating carbon nanotube-catalyst adhesion strengths. Phys. Rev. B 2007, 75, 115419.

[65] Zhu, W.; Rosen, A.; Bolton, K. Changes in single-walled carbon nanotube chirality during growth and regrowth. J. Chem. Phys. 2008, 128, 124708. 